Series A

I. MATHEMATICA

421

\title{
SOME REMARKS ON THE VALUE DISTRIBUTION OF ENTIRE FUNCTIONS
}

$\mathrm{BY}$

SAKARI TOPPILA 
Communicated 9 February 1968 by K. I. Virtanen and Lauri Myrberg 


\section{Introduction}

1. We call a set $E$ a Picard set for entire functions if every entire nonrational function $f$ omits at most one finite value in $-E$.

Lehto [2] has proved that a countable set

$$
E=\{\infty\} \cup\left\{a_{n}\right\}_{n=1,2, \ldots}
$$

whose points converge to infinity is a Picard set for entire functions if the points $a_{n}$ satisfy the condition

$$
\left|a_{n}\right| a_{n+1} \mid=O\left(n^{-2}\right) .
$$

Matsumoto [4] has proved the same assertion under the condition

$$
\log \left|a_{n+1}\right| a_{n} \mid \geq M(n),
$$

where $M(n)$ are positive numbers such that

$$
\limsup _{n \rightarrow \infty} \frac{K^{1 / M(n)}}{M(1)+M(2)+\ldots+M(n)}<\infty
$$

( $K$ a positive constant). In this paper we prove that there exist Picard sets for entire functions, which contain a sequence of discs converging to the point at infinity.

Winkler [7] has among other things proved that the entire functions

$$
w(z)=\prod_{n=1}^{\infty}\left(1-z / a_{n}\right)
$$

with $\left|a_{n+1}\right| a_{n} \mid \geq q>1$ take any finite value a infinitely often in the union of the discs

$$
D_{n}=\left\{z:\left|z-a_{n}\right|<\varrho_{n}\right\}
$$

with $\varrho_{n}=\varepsilon\left|a_{n}\right|^{-p}$ for any $\varepsilon>0$ and $p>0$, and that they take any value only finitely often in the complement of this union (See also Lehto [3], Theorem 4). Our Theorem 1 shows that the same is not true if the radii $\varrho_{n}$ of $D_{n}$ satisfy the condition $\left|a_{n}\right|=o\left(-\log \varrho_{n}\right)$. 


\section{Picard sets for entire functions}

2. We begin by presenting three lemmas. We denote $\log ^{+} \delta=$ $\max \{0, \log \delta\}$ for $\delta \geq 0$. Our first lemma is a consequence of Schottky's theorem which is proved by Ahlfors in the following form (Dinghas [1], p. 294):

If $g(z)$ is regular in $|z|<1$ and $g(z) \neq 0,1$ there, then

$$
\stackrel{+}{\log }|g(z)| \leq \frac{1+|z|}{1-|z|}(7+\log |g(0)|) .
$$

Lemma 1. Let $f$ be analytic in an annulus $r<|z|<R \quad(0<r<$ $R<\infty)$ and omit the values 0 and 1 there. 'Then

$$
\log \left(\max _{\mid z=\sqrt{r R}}|f(z)|\right) \leq\left\{7+\log _{|z|=\sqrt{r R}}^{+}\left(\min _{\sqrt{r R}}|f(z)|\right)\right\} \exp \left\{\frac{\pi^{2}}{\log (R / r)}\right\} .
$$

Proof. We choose $z_{0}$ such that $\left|z_{0}\right|=\sqrt{r R}$ and $\left|f\left(z_{0}\right)\right|=\min _{z=\sqrt{r R}}|f(z)|$. We denote $\mu=\log (R / r)$. The composite function $g(\zeta)=f\left(z_{0} \sqrt{r / R} e^{\xi}\right)$ is regular, different from 0 and 1 , and has the period $2 \pi i$ in the strip domain

$$
D=\{\zeta: 0<\operatorname{Re} \zeta<\mu,-\infty<\operatorname{Im} \zeta<+\infty\} \text {. }
$$

Hence any value taken by $f$ on $|z|=\sqrt{r R}$ is taken by $g$ on the segment

$$
I=\{\zeta: \operatorname{Re} \zeta=\mu / 2,-\pi \leq \operatorname{Im} \zeta \leq \pi\} .
$$

Especially $g(\mu / 2)=f\left(z_{0}\right)$. The function

$$
w(\zeta)=\frac{e^{-\pi i-/ \mu}-i}{e^{-\pi i \zeta / \mu}+i}
$$

maps $D$ onto the unit disc $w<1$ conformally and

$$
w(I)=\left\{w:-\frac{e^{\pi^{2} / \mu}-1}{e^{\pi^{2} / \mu}+1} \leq \operatorname{Re} w \leq \frac{e^{\pi^{2} / \mu}-1}{e^{\pi^{2} \mu}+1}, \operatorname{Im} w=0\right\} .
$$

Since $w(\mu / 2)=0$ and $g(\mu / 2)=f\left(z_{0}\right)$, the lemma follows from Schottky's theorem.

Let $\Sigma$ be the Riemann sphere with radius $1 / 2$ touching the $w$-plane at the origin. The chordal distance of the images on $\Sigma$ of two points $w$ and $w^{\prime}$ in the plane is denoted by $\left[w, w^{\prime}\right]$, and $C(w, \delta)$ is the spherical open disc with centre at the image of $w$ and with chordal radius $\delta$. The following lemma is proved by Matsumoto [5].

Lemma 2. Let $f$ be analytic in an annulus $1<|z|<e^{\mu}$ and omit the values 0 and 1 . There exists a positive constant $A$ such that the 
spherical diameter of the image curve of $|z|=e^{u / 2}$ by $f$ is not greater than $A e^{-\mu / 2}$ for all $\mu>0$.

Let $\Delta$ be a triply connected domain with boundary components $\Gamma_{1}$, $\Gamma_{2}$ and $\Gamma_{3}$, and let $f$ be analytic and omit the values 0 and 1 in $\bar{A}$. We assume that the images of $\Gamma_{1}, \Gamma_{2}$ and $\Gamma_{3}$ by $f$ are contained in the spherical discs $C_{1}, C_{2}$ and $C_{3}$, respectively, and give the following lemma of Matsumoto [5].

Lemma 3. Let $\delta>0$ be so small that the spherical discs $C(0,2 \delta)$, $C(1,2 \delta)$ and $C(\infty, 2 \delta)$ are mutually disjoint. If the radii of $C_{1}, C_{2}$ and $C_{3}$ are less than $\delta / 2$, only two possibilities can occur:

(1) $C_{1}, C_{2}$ and $C_{3}$ contain the origin, the point $w=1$, and the point at infinity, one by one, so that $C_{1}, C_{2}$ and $C_{3}$ are contained in $C(0, \delta)$, $C(1, \delta)$ and $C(\infty, \delta)$, respectively, and $f$ takes each value outside the union of $C(0, \delta), C(1, \delta)$ and $C(\infty, \delta)$ once and only once in $\Delta$.

(2) Of $C_{1}, C_{2}$ and $C_{3}$ none can be disjoint from the union of the other two, so that there is a disc with radius less than $3 \delta / 2$ which contains the image of $\Delta$.

3. We consider first a sequence of discs with the middle points lying in a half plane.

Theorem 1. Let $D_{n}, n=1,2, \ldots$, be a sequence of discs with centre $z_{n}, \operatorname{Re} z_{n}>1$, and with radius $\varrho_{n}$. If

$$
\left|z_{n+1}\right| z_{n} \mid>\alpha>1
$$

for $n=1,2, \ldots$, and

$$
\left|z_{n}\right|=o\left(-\log \varrho_{n}\right)
$$

then $E=\{\infty\} \cup \bigcup_{n=1}^{\infty} D_{n}$ is a Picard set for entire functions.

Proof. It is obviously sufficient to prove that the assumption of the existence of a function $f$, analytic and non-rational for $z \neq \infty$, and different from 0 and 1 outside $E$ leads to a contradiction. There is no loss of generality to assume that each $D_{n}$ contains at least one zero or 1-point of $f$, for we can delete from $\left\{D_{n}\right\}$ all other dises and the remaining discs also satisfy conditions (1) and (2).

We consider the function $g(z)=f(1 / z) . g$ is analytic and non-rational for $z \neq 0$. Since $\lim \varrho_{n}=0$, we can take $M>0$ such that the set

$$
\{z:|z|>M, \operatorname{Re} z<0\}
$$

contains no point of $E-\{\infty\}$. Then by (1) and (2), there exist $0<\varrho_{0}<$ $1 / M$ and a sequence of dises $B_{n}, n=1,2, \ldots$, with centre $s_{n}$, Re $s_{n}>0$, and with radius $\sigma_{n}$ satisfying the conditions 
(3)

$$
\left|s_{n} / s_{n+1}\right|>\alpha>1
$$

for $n=1,2, \ldots$, and

$$
1 /\left(-\log \sigma_{n}\right)=o\left(\left|s_{n}\right|\right),
$$

such that $\bigcup_{n=1}^{\infty} B_{n} \subset\{z: \operatorname{Re} z>0\}, g(z) \neq 0,1$ outside $F=\{0\} \cup \bigcup_{n=0}^{\infty} B_{n}$, where $B_{0}=\left\{z:|z|>\varrho_{0}\right\}$, and each $B_{n}$ contains at least one zero or 1-point of $g$.

By (3) and (4), we can choose an $n_{1}$ so large that the annulus

$$
S_{n}=\left\{z: \sigma_{n}<\left|z-s_{n}\right|<\left|s_{n}\right|(\alpha-1) / 2 \alpha\right\}
$$

contains no point of $F$ for any $n \geq n_{1}$. Applying Lemma 2 to $S_{n}$ we conclude that the spherical diameter of the image of

$$
\gamma_{n}=\left\{z:\left|z-s_{n}\right|=\sqrt{\sigma_{n}\left|s_{n}\right|(\alpha-1) / 2 \alpha}\right\}
$$

by $g$ is dominated by

$$
\delta_{n}=A \sqrt{2 \alpha \sigma_{n} /(\alpha-1)\left|s_{n}\right|}
$$

for $n \geq n_{1}$. Hence there exists a spherical disc $C_{n}$ with radius less than $\delta_{n}$ which contains this image.

We take $\delta>0$ so small that the discs $C(0,2 \delta), C(1,2 \delta)$ and $C(\infty, 2 \delta)$ are mutually disjoint. Since the origin is an essential singularity of $g$, we have

$$
\lim _{r \rightarrow 0} M(r)=\infty,
$$

where $M(r)=\max \{|g(z)|:|z|=r\}$.

By (3) and (4), we can take an $n_{2} \geq n_{1}$ such that the annulus

$$
R_{n}=\left\{z:\left|s_{n}\right|(2 \alpha /(3 \alpha-1))^{2}<|z|<2 \alpha \mid s_{n} /(3 \alpha-1)\right\}
$$

contains no point of $F$ for $n \geq n_{2}$. The modulus of each $R_{n}$ is $\log ((3 \alpha-1) / 2 \alpha)>0$. Applying Lemma 1 to $R_{n}, n \geq n_{2}$, we see by $(6)$ that the image of

$$
\lambda_{n}=\left\{z:|z|=\left|s_{n}\right|(2 \alpha /(3 \alpha-1))^{3 / 2}\right\}
$$

by $g$ is contained in $C(\infty, \delta / 2)$ for sufficiently large $n$, say for $n \geq n_{3}$. We may assume $n_{3} \geq n_{2}$.

By (4) there exists $n_{4} \geq n_{3}$ such that $\delta_{n}<\delta / 4$ for $n \geq n_{4}$. Applying Lemma 3 to the triply connected domain with $\lambda_{n}, \lambda_{n-1}$ and $\gamma_{n}$ as boundary, we see that $C_{n}$ is contained in $C(\infty, \delta)$ for $n>n_{4}$.

We choose $n_{5}>n_{4}$ so large that $\left|s_{n}\right|<\varrho_{0} / 2$ for $n \geq n_{5}$. We apply Schottky's theorem to the disc 


$$
\left\{z:\left|z+\varrho_{0} / 2\right|<\varrho_{0} / 2\right\}
$$

and get

$\log ^{+}\left|g\left(-\left|s_{n}\right|\{2 \alpha /(3 \alpha-1)\}^{3 / 2}\right)\right| \leq\left\{7+\log \left|g\left(-\varrho_{0} / 2\right)\right|\right\} \varrho_{0}\left|s_{n}\right|^{-1}\{(3 \alpha-1) / 2 \alpha\}^{3 / 2}$ for $n \geq n_{5}$. We use $K_{1}$ and $K_{2}$ to denote positive constants depending only on $\varrho_{0}, \log \left|g\left(-\varrho_{0} / 2\right)\right|$ and $\alpha$. Applying Lemma 1 to the annulus $R_{n}$ we get

$$
\log |g(z)|<K_{1}+K_{2}\left|s_{n}\right|^{-1}=M_{n}
$$

for $z \in \lambda_{n}$.

We denote by $\Lambda_{n}$ the unbounded component of the complement of $\lambda_{n}$.

The maximum principle applied to $\Lambda_{n}$ yields $\log |g(z)|<M_{n}$ in $\Lambda_{n}$. We take $n_{6} \geq n_{5}$ so large that

$$
\left|s_{n}\right|-\sqrt{\sigma_{n}\left|s_{n}\right|(\alpha-1) / 2 \alpha>\left|s_{n}\right|(2 \alpha /(3 \alpha-1))^{3 / 2}}
$$

for any $n \geq n_{6}$. Then we have $\gamma_{n} \subset \Lambda_{n}$, and $g\left(\gamma_{n}\right) \subset T_{n}$ with

$$
T_{n}=\left\{w:[w, \infty] \geq\left(1+e^{2 M_{n}}\right)^{-1 / 2}\right\} .
$$

Since $g\left(\gamma_{n}\right) \subset C_{n}$, we get $C_{n} \cap T_{n} \neq \varnothing$.

Instead of (4) we can write

$$
\left|s_{n}\right|^{-1}=o\left(-\log \sigma_{n}\right),
$$

and this implies by (5) and (7) that there exists $n_{7} \geq n_{6}$ such that

$$
\delta_{n}<1 / 4\left(1+e^{2 M_{n}}\right)^{1 / 2}
$$

for any $n \geq n_{7}$.

Since $C_{n} \cap T_{n} \neq \varnothing$, we see by (8) that $C_{n}$ cannot contain the point at infinity for $n \geq n_{7}$. Then the maximum principle applied to the bounded disc $G_{n}$ with $\gamma_{n}$ as boundary yields $g\left(G_{n}\right) \subset C_{n}$. Since $B_{n} \subset G_{n}$, we get $g\left(B_{n}\right) \subset C_{n}$. This is a contradiction, for $C_{n}$ contains no zero or 1-point of $g$, and the theorem is proved.

4. If we assume that the middle points of the discs $D_{n}$ need not lie in a half plane, we must replace the condition (2) by a stronger one.

Theorem 2. Let $D_{n}, n=1,2, \ldots$, be a sequence of discs with centre $z_{n}$ and with radius $\varrho_{n}$. If

$$
\left|z_{n+1}\right| z_{n} \mid>\alpha>1
$$

for $n=1,2, \ldots$, and 


$$
\left|z_{n}\right|^{n}=O\left(-\log \varrho_{n}\right)
$$

the $E=\{\infty\} \cup \bigcup_{n=1}^{\infty} D_{n}$ is a Picard set for entire functions.

Proof. As in the proof of Theorem 1, it is sufficient to prove that the assumption of the existence of a function $f$, analytic and non-rational for $z \neq \infty$, and different from 0 and 1 outside $E$, leads to a contradiction.

We consider the function $g(z)=f(1 / z) . g$ is analytic and non-rational for $z \neq 0$. By (a) and (b), there exist $\varrho_{0}>0$ and a sequence of discs $B_{n}$, $n=1,2, \ldots$, with centre $t_{n}$ and with radius $\sigma_{n}$ satisfying the conditions

$$
\left|t_{n}\right| t_{n+1} \mid>\alpha>1
$$

for $n=1,2, \ldots$, and

$$
1 /\left(-\log \sigma_{n}\right)=O\left(\left|t_{n}\right|^{n}\right),
$$

such that $g(z) \neq 0,1$ outside $F=\{0\} \cup \bigcup_{n=0}^{\infty} B_{n}$, where $B_{0}=\left\{z:|z|>\varrho_{0}\right\}$, and each $B_{n}$ contains at least one zero or 1-point of $g$.

We take $\delta>0$ so small that the spherical disc $C(0,2 \delta), C(1,2 \delta)$ and $C(\infty, 2 \delta)$ are mutually disjoint. As in the proof of Theorem 1, we can take $n_{1}$ so large that for any $n \geq n_{1}$ the image of

$$
\gamma_{n}=\left\{z:\left|z-t_{n}\right|=\sqrt{\sigma_{n}\left|t_{n}\right|(\alpha-1) / 2 \alpha}\right\}
$$

by $g$ is contained in a spherical disc $C_{n}$ with radius less than

$$
\delta_{n}=A \sqrt{2 \alpha \sigma_{n} /(\alpha-1)\left|t_{n}\right|},
$$

where $A$ is the constant of Lemma 2, and $C_{n} \subset C(\infty, \delta)$.

We choose $n_{2} \geq n_{1}$ so large that

$$
\begin{gathered}
2 d=\left|t_{n_{2}}\right|<\min \left\{\varrho_{0},(2 \alpha /(3 \alpha-1))^{3 / 2}\right\}, \\
\sum_{n=n_{2}}^{\infty} \sigma_{n} /\left|t_{n}\right|<1 / 8,
\end{gathered}
$$

and that for any $n \geq n_{2}$, the annulus

$$
R_{n}=\left\{z:\left|t_{n}\right|(2 \alpha /(3 \alpha-1))^{2}<|z|<2 \alpha\left|t_{n}\right| /(3 \alpha-1)\right\}
$$

contains no point of $F$. We denote $L=\max \{\log |g(z)|:|z|=d\}$. We take $n_{3} \geq n_{2}$ such that $\left|t_{n_{3}}\right|<d$. Then we see by (g) that there exists for each $n \geq n_{3}$ a $\varphi_{n}$ such that the set

$$
\left\{z:\left|t_{n}\right|(2 \alpha /(3 \alpha-1))^{2}<|z|<2 d,\left|\arg z-\varphi_{n}\right|<\pi / 2 n\right\}
$$

contains no point of $F$. Considering the function $h(\zeta)=g(\sqrt[n]{\zeta})$ on the disc 


$$
\left\{\zeta:\left|\zeta-d^{n} e^{i n \varphi_{n}}\right|<d^{n}-\left|t_{n}\right|^{n}(2 \alpha /(3 \alpha-1))^{2 n}\right\}
$$

we get by Schottky's theorem

$$
\begin{aligned}
N_{n} & =\log \left|g\left(\left|t_{n}\right|\{2 \alpha /(3 \alpha-1)\}^{3 / 2} e^{i r_{n}}\right)\right| \\
& \leq \frac{2 d^{n}(7+L)}{\left(\left|t_{n}\right|\{2 \alpha /(3 \alpha-1)\}^{3 / 2}\right)^{n}-\left(\left|t_{n}\right|\{2 \alpha /(3 \alpha-1)\}^{2}\right)^{n}} .
\end{aligned}
$$

We take $n_{4} \geq n_{3}$ such that

$$
N_{n} \leq 4 d^{n}(7+L)\left(\left|t_{n}\right|\{2 \alpha /(3 \alpha-1)\}^{3 / 2}\right)^{-n}
$$

for $n \geq n_{4}$. We apply Lemma 1 to $R_{n}$ and get

$$
\log |g(z)| \leq\left(7+N_{n}\right) \exp \left\{\pi^{2} / \log ((3 \alpha-1) / 2 \alpha)\right\}
$$

for $z \in \lambda_{n}$ with

$$
\lambda_{n}=\left\{z:|z|=\left|t_{n}\right|(2 \alpha /(3 \alpha-1))^{3 / 2}\right\} .
$$

By the conditions (f), (h) and (i), we get for $z \in \lambda_{n}$ the estimate

$$
\log |g(z)| \leq K_{1}+K_{2}\left(2\left|t_{n}\right|\right)^{-n}=M_{n},
$$

where $K_{1}$ and $K_{2}$ are positive constants depending only on $L$ and $\alpha$.

We denote by $\Lambda_{n}$ the unbounded component of the complement of $\lambda_{n}$.

The maximum principle applied to $\Lambda_{n}$ yields $\log ^{+}|g(z)| \leq M_{n}$ in $\Lambda_{n}$. We take $n_{5} \geq n_{4}$ so large that $\gamma_{n} \subset \Lambda_{n}$ and $\gamma_{n} \cap F=\emptyset$ for $n \geq n_{5}$. Then we have $g\left(\gamma_{n}\right) \subset T_{n}$ with

$$
T_{n}=\left\{w:[w, \infty] \geq\left(1+e^{2 M_{n}}\right)^{-1 / 2}\right\},
$$

and $C_{n} \cap T_{n} \neq \varnothing$. We get by (d)

$$
\left|t_{n}\right|^{-n}=O\left(-\log \sigma_{n}\right)
$$

and this implies by (e) and (j) that there exists $n_{6} \geq n_{5}$ such that

$$
\delta_{n}<1 / 4\left(1+e^{2 M_{n}}\right)^{1 / 2}
$$

For any $n \geq n_{6}$.

Since $C_{n} \cap T_{n} \neq \varnothing$, we see by (k) that $C_{n}$ cannot contain the point at infinity for $n \geq n_{6}$. Then the maximum principle applied to the bounded disc $G_{n}$ with $\gamma_{n}$ as boundary yields $g\left(G_{n}\right) \subset C_{n}$. Since $B_{n} \subset G_{n}$, we get $g\left(B_{n}\right) \subset C_{n}$. This is a contradiction, for $C_{n}$ contains no zero or 1-point of $g$, and the theorem is proved. 


\section{Meromorphic functions}

5. No theorem like the theorems 1 and 2 is valid for meromorphic functions. We can in fact prove that given any sequence of discs $D_{n}, \quad n=1,2, \ldots$, which converge to the point at infinity, then there exists a function $f$, meromorphic and non-rational for $z \neq \infty$, and bounded outside

$$
E=\{\infty\} \cup \bigcup_{n=1}^{\infty} D_{n}
$$

We construct a sequence $B_{n}, n=1,2, \ldots$, of discs with centre $z_{n}$ and with radius $\varrho_{n}$ satisfying the conditions $\left|z_{1}\right|>1$, and $B_{n} \subset \bigcup_{p=1}^{\infty} D_{p}$,

$$
\left|z_{n+1}\right| z_{n} \mid>e^{n}
$$

and

$$
\varrho_{n} /\left|z_{n}\right|<e^{-n}
$$

for $n=1,2, \ldots$ We denote $r_{n}=\varrho_{n} e^{-n}$, and define

$$
g(z)=\prod_{n=1}^{\infty} \frac{1-z /\left(z_{n}-r_{n}\right)}{1-z /\left(z_{n}+r_{n}\right)} .
$$

For $z \notin B_{n}$ we get by (1) and (2)

$$
\left|\frac{1-z /\left(z_{n}-r_{n}\right)}{1-z /\left(z_{n}+r_{n}\right)}\right| \leq 1+16 r_{n} / \varrho_{n}=1+16 e^{-n} .
$$

Then we have for $z \notin\{\infty\} \cup \bigcup_{n=1}^{\infty} B_{n}$ the estimate

$$
|g(z)| \leq \prod_{n=1}^{\infty}\left(1+16 e^{-n}\right)<\infty
$$

University of Helsinki and University of Jyväskylä

Finland 


\section{References}

[1] Dinghas, A.: Vorlesungen über Funktionentheorie. - Springer-Verlag. BerlinGöttingen-Heidelberg, 1961.

[2] Lehto, O.: A generalization of Picard's theorem. - Ark. Mat. 3 (1958), 495-500.

[3] - - The spherical derivative of meromorphic functions in the neighbourhood of an isolated singularity. - Comment. Math. Helv. 33 (1959), 196-205.

[4] Matsumoto, K.: Remark on Lehto's paper: "A generalization of Picard's theorem.» Proc. Japan. Acad. 38 (1962), 636-640.

[5] - - - Some remarks on Picard sets. - Ann. Acad. Sci. Fenn. A I 403, 1967.

[6] Toppila, S.: Picard sets for meromorphic functions. - Ann. Acad. Sci. Fenn. A I 417. 1967.

[7] Winkler, J.: Zur Verteilung der a-Stellen spezieller ganzer Funktionen. - Math. Z. 101 (1967), 143-151. 